\title{
Caracterización del método SVPWM con inversor trifásico de dos niveles
}

\author{
Juan Tisza ${ }^{1,2}$, Javier Villegas ${ }^{2}$ \\ ${ }^{1}$ Universidad Nacional de Ingeniería, Av. Túpac Amaru 210, Rímac, Lima Perú \\ 'Universidad Nacional Mayor de San Marcos, Ciudad Universitaria, Lima, Perú \\ Recibido 17 de junio del 2019, Revisado el 17 de julio de 2019 \\ Aceptado el 19 de julio de 2019
}

DOI: https://doi.org/10.33017/RevECIPeru2019.0005/

\section{Resumen}

Las cargas en Corriente Alterna (CA) requieren voltaje variable y frecuencia variable. Estos requisitos se cumplen con un inversor de fuente de voltaje (VSI). Se puede lograr un voltaje de salida variable variando la tensión de CC de entrada y manteniendo constante la ganancia del inversor. Por otro lado, si la tensión de entrada CC es fija y no es controlable, se puede lograr una tensión de salida variable variando la ganancia del inversor, lo que normalmente se logra mediante el control de modulación por ancho de pulso dentro del inversor. Hay varias técnicas de modulación de ancho de pulso, pero la técnica de vector espacial es una buena opción entre todas las técnicas para controlar el inversor de fuente de voltaje. La modulación por ancho de pulso de vector espacial (SVPWM) es un método avanzado y muy popular con varias ventajas tales como la utilización efectiva del bus de CC, menos generación de armónicos en voltaje de salida, menos pérdidas de conmutación, amplio rango de modulación lineal, etc. En este documento, se ha tomado un inversor de fuente de voltaje constante CC y se ha implementado la SVPWM para VSI de dos niveles utilizando MATLAB / SIMULINK.

Descriptores: Modulación de ancho de pulso (PWM), modulación de ancho de pulso de vector espacial (SVPWM), distorsión armónica total (THD), inversor de fuente de voltaje (VSI).

\section{Abstract}

Alternating Current (AC) loads require variable voltage and variable frequency. These requirements are met by a voltage supply inverter (VSI). A variable output voltage can be achieved by varying the input DC voltage and keeping the inverter gain constant. On the other hand, if the DC input voltage is fixed and not controllable, a variable output voltage can be achieved by varying the gain of the inverter, which is normally achieved by controlling the pulse width modulation within the inverter. There are several pulse width modulation techniques, but the spatial vector technique is a good choice among all the techniques for controlling the voltage source inverter. Spatial vector pulse width modulation (SVPWM) is an advanced and very popular method with several advantages such as effective utilization of CC bus, less harmonic generation in output voltage, less switching losses, wide range of linear modulation, etc. In this document, a CC constant voltage source inverter has been taken and SVPWM has been implemented for two-level VSI using MATLAB / SIMULINK.

Keywords: Pulse Width Modulation (PWM), Space Vector Pulse Width Modulation (SVPWM), Total Harmonic Distortion (THD), Voltage Source Inverter (VSI).

\section{Introducción}

En este artículo se caracteriza, mediante la simulación de una aplicación que cumple con el requerimiento de los variadores de CA que buscan un suministro de frecuencia variable y voltaje variable de alta potencia. Debido a muchos avances en dispositivos de energía de estado sólido, los motores de inducción de CA de velocidad variable alimentados por convertidores de potencia de conmutación son cada vez más populares. Para regular la frecuencia y el voltaje de un motor, 
generalmente se usan convertidores de potencia de conmutación porque proporciona mayor eficiencia y rendimiento con menos ruido [3].

En los últimos años, se han propuesto muchas técnicas de PWM para lograr los siguientes objetivos; menos distorsión armónica total, menos pérdidas de conmutación, amplio rango de modulación lineal, menos tiempo de cálculo, fácil de implementar y control preciso también. Además, las técnicas de PWM se utilizan para controlar la tensión de salida de un inversor. La principal ventaja de PWM es que la pérdida de potencia en los dispositivos de conmutación es muy baja. PWM también funciona bien con controles digitales debido a su naturaleza de encendido/apagado y puede configurar fácilmente el ciclo de trabajo necesario [1]. Hay varias técnicas de modulación, como modulación de ancho de pulso sinusoidal (SPWM), modulación trapezoidal, modulación de escalera, modulación escalonada, eliminación selectiva de armónicos, control de vector espacial, inyección de Tercer armónico, modulación de ancho de pulso de vector espacial (SVPWM), etc. La técnica vectorial es la mejor entre todas las técnicas porque tiene varias ventajas, como la utilización efectiva de la tensión CC, menos distorsión armónica en la tensión/corriente de salida, cálculo digital simple para el tiempo de conmutación, etc [2].

\section{Principios de la Modulación por Ancho de Pulso del Vector Espacial (SVPWM)}

\subsection{Inversor VSI de dos niveles}

En la Fig. 1 muestra el inversor de fuente de voltaje (VSI) de dos niveles y tres patas de tres fases. Tiene seis interruptores de potencia (S1 a S6) que se utilizan para dar forma a la tensión de salida. Los interruptores S1, S3, S5 son interruptores superiores y los interruptores S2, S4, S6 son interruptores inferiores. En cada rama, debe haber dos interruptores y estos dos interruptores se complementan entre sí. Si S1 y S4 son los interruptores en la primera etapa de VSI trifásico y cuando S1 está activado, es decir, S1 = 1, S4 debería estar desactivado, es decir, $S 4=0 \mathrm{y}$ viceversa. Por lo tanto, el control de los interruptores se realiza de forma que no se activen dos interruptores al mismo tiempo en una misma rama, de lo contrario, el tramo se cortocircuitará y, por lo tanto, habrá una tensión de salida cero en el lado de la carga.

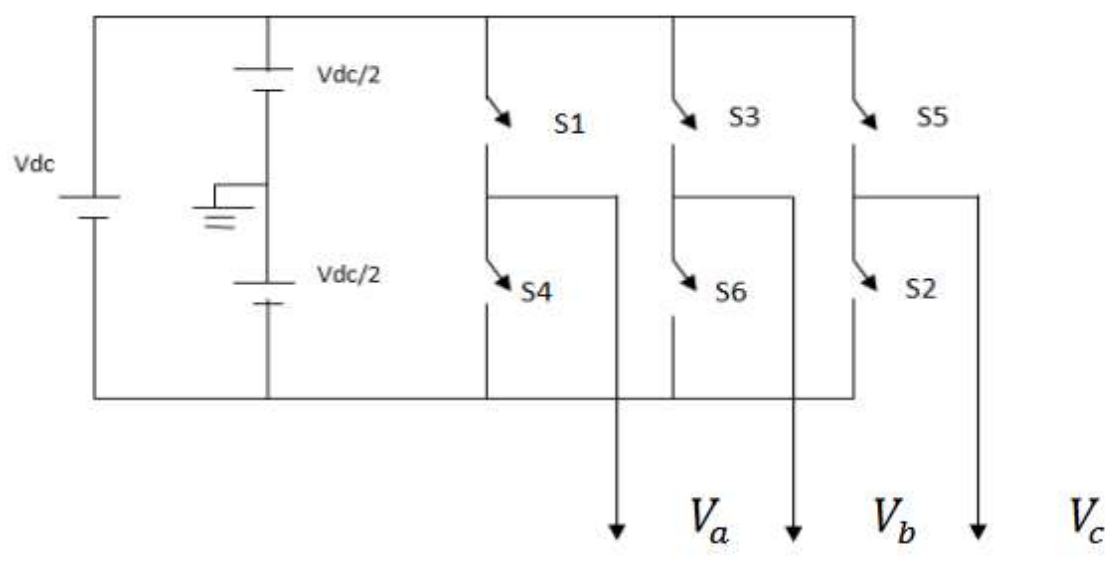

Figura 1: Inversor trifásico de dos niveles.

\subsection{Modulación SVPWM}

La modulación del ancho de pulso de vector espacial (SVPWM) está basada en el hecho que solo existe dos variables independientes en un sistema trifásico (tensión y frecuencia). Se pueden usar coordenadas ortogonales para representar una tensión trifásica en el plano complejo. La tensión trifásica puede representarse, mediante la transformación de Clarke, en un plano bidimensional como:

$$
\left[\begin{array}{l}
V_{\alpha} \\
V_{\beta}
\end{array}\right]=\frac{2}{3}\left[\begin{array}{lll}
1 & -\frac{1}{2} & -\frac{1}{2} \\
0 & \frac{\sqrt{3}}{2} & -\frac{\sqrt{3}}{2}
\end{array}\right]\left[\begin{array}{l}
V_{a} \\
V_{b} \\
V_{c}
\end{array}\right]
$$

En la técnica de SVPWM, la tensión trifásica de salida es representada mediante un vector de referencia, que rota a una frecuencia angular de $\omega=2 \pi f$. La modulación SVPWM se encarga de aproximar este vector de referencia a través de los 
estados de conmutación presentes en el inversor

[1].

Tabla 1: Estados de conmutación del inversor de dos niveles.

\begin{tabular}{|l|r|r|}
\hline Vector Espacial & Estados de conmutación & Interruptores activos \\
\hline \multirow{2}{*}{ Vector nulo } & $\mathrm{V}_{7}(111)$ & $1,3,5$ \\
\cline { 2 - 3 } & $\mathrm{V}_{0}(000)$ & $4,6,2$ \\
\hline \multirow{3}{*}{ Vector activo } & $\mathrm{V}_{1}(100)$ & $4,6,5$ \\
\cline { 2 - 3 } & $\mathrm{V}_{2}(110)$ & $4,3,2$ \\
\cline { 2 - 3 } & $\mathrm{V}_{3}(010)$ & $4,3,5$ \\
\cline { 2 - 3 } & $\mathrm{V}_{4}(011)$ & $1,6,2$ \\
\cline { 2 - 3 } & $\mathrm{V}_{5}(001)$ & $1,6,5$ \\
\hline
\end{tabular}

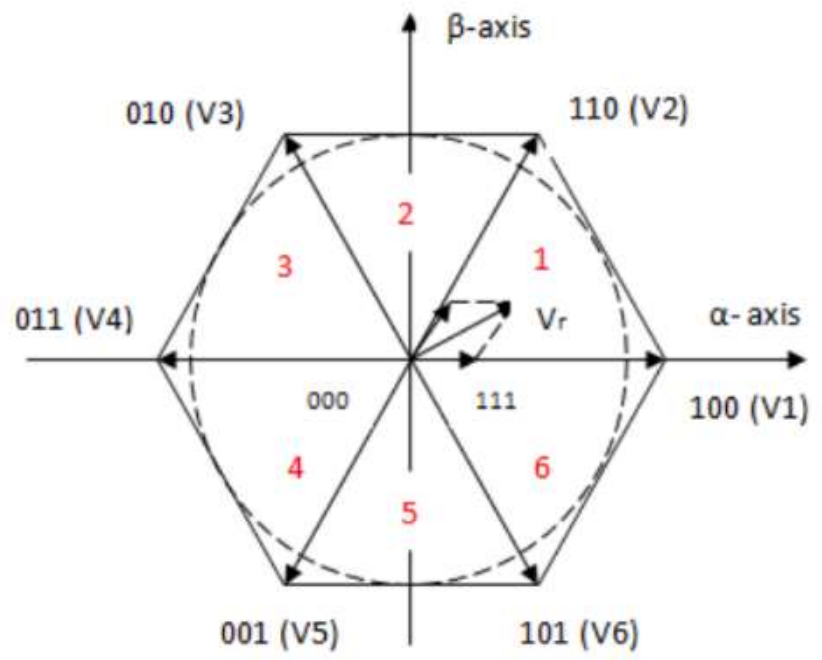

Figura 2: Vectores espaciales y sectores de conmutación para un inversor de dos niveles.

Los ocho posibles estados de conmutación del inversor son representados como dos vectores nulos y seis vectores activos como se muestra en la Tabla 1. El vector de referencia, que nos ayudará a controlar la tensión trifásica en la salida, esta expresado de la siguiente manera:

$$
V_{r}=\frac{2 m}{3}\left[\begin{array}{ccc}
1 & -\frac{1}{2} & -\frac{1}{2} \\
0 & \frac{\sqrt{3}}{2} & -\frac{\sqrt{3}}{2}
\end{array}\right]\left[\begin{array}{l}
V_{a} \\
V_{b} \\
V_{c}
\end{array}\right]
$$

En donde $m$ es el índice de modulación, reemplazando las tensiones de línea obtenemos una versión más compacta del vector de referencia:

$$
V_{r}=\left[\begin{array}{l}
m V_{L} \cos \omega t \\
m V_{L} \sin \omega t
\end{array}\right]
$$

En donde $\omega$ es la frecuencia angular fundamental del sistema trifásico. En la Fig. 2 se muestra el vector de referencia $V_{r}$ y los vectores espaciales de cada estado de conmutación. El vector de referencia puede ser aproximado en cada ciclo de conmutación mediante el cambio entre los vectores activos adyacentes y los vectores nulos, como se muestra en la Fig. 3. Para mantener la frecuencia de conmutación efectiva a valor mínimo, la secuencia de conmutación entre estos vectores está organizada de tal manera que solo una rama se ve afectada en cada paso.

En la Tabla 2 se muestran los distintos sectores de conmutación respecto a la posición del vector de referencia. Según el sector en que se encuentre el vector de referencia, existen distintos patrones de conmutación que se irán activando. Como resultado, cuando el vector de referencia complete una revolución en el espacio, la tensión de salida del inversor varia un ciclo en el tiempo.

Tabla 2: Sectores de conmutación y sus ángulos respectivos.

\begin{tabular}{|l|r|}
\hline Sectores & Posición $\mathrm{Vr}$ \\
\hline 1 & $0<\mathrm{wt}<60$ \\
\hline 2 & $60<\mathrm{wt}<120$ \\
\hline 3 & $120<\mathrm{wt}<180$ \\
\hline 5 & $180<\mathrm{wt}<240$ \\
\hline
\end{tabular}




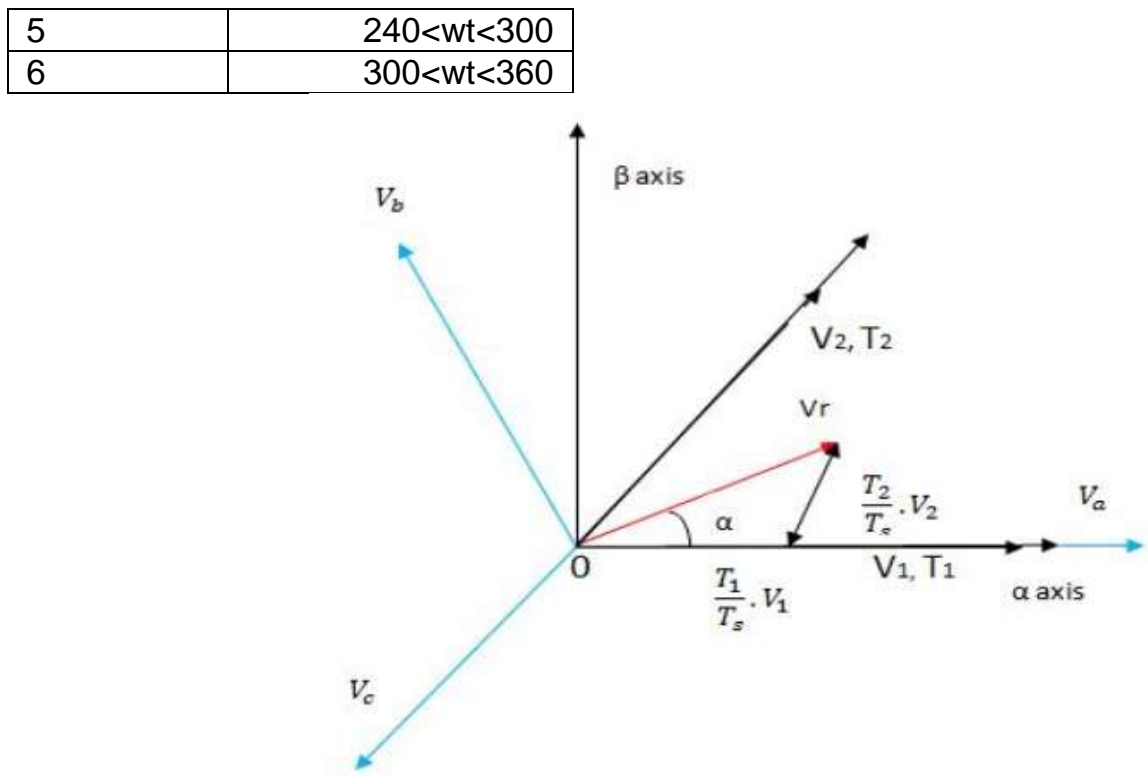

Figura 3: Aproximación del vector de referencia en el sector 1.

\subsection{Cálculo del Tiempo de Aplicación de los Vectores Espaciales}

Como hemos visto antes, el vector de referencia se puede aproximar mediante la combinación de los vectores activos adyacentes y uno o dos vectores nulos. Consideremos que el vector de referencia se encuentra en el sector $k$ y sea $T_{s}$ el periodo de conmutación, $V_{k}$ es aplicado por un tiempo $T_{1}, V_{k+1}$ es aplicado por $T_{2}$ y $V_{0-7}$ es aplicado por $T_{s}-T_{1}-T_{2}$.

El valor medio del voltaje de referencia en un periodo de conmutación se aproxima de la siguiente manera:

$\int_{0}^{T_{s}} V_{r}=\int_{0}^{T_{1}} V_{k} d t+\int_{T_{1}}^{T_{1}+T_{2}} V_{k+1} d t+\int_{T_{1}+T_{2}}^{T_{s}} V_{0-7} d t$

Considerando que el vector de referencia se mantiene constante en un periodo de conmutación (esto significa que el periodo de conmutación Ts es pequeño en relación al periodo de la línea trifásica), la ecuación 4 se reduce a [2]:

$$
T_{s} V_{r}=T_{1} V_{k}+T_{2} V_{k+1}
$$

En la Fig. 3 se muestra esto gráficamente para el sector 1. Resolviendo la ecuación 5 calculamos los tiempos $T_{k}$ y $T_{k+1}$ para cada sector como sigue:

$$
\begin{aligned}
& T_{1}=T_{S} \cdot m \cdot \frac{\sin \left(k \frac{\pi}{3}-\alpha\right)}{\sin \left(\frac{\pi}{3}\right)} \\
& T_{2}=T_{S} \cdot m \cdot \frac{\sin \left(\alpha-(k-1) \frac{\pi}{3}\right)}{\sin \left(\frac{\pi}{3}\right)}
\end{aligned}
$$

$$
T_{0}=T_{S}-T_{1}-T_{2}
$$

El índice de modulación $m$ se elige de la siguiente manera:

$$
m=\frac{\left|V_{r}\right|}{V_{L_{m a ́ x}}}
$$

En donde $V_{L_{\max }}$ es la tensión máxima de línea que puede generar el inversor, en nuestro caso :

$$
V_{L_{\text {máx }}}=\frac{2}{3} V_{d c}
$$

La división de los sectores y el tiempo de aplicación de los vectores activos son iguales para todas las estrategias de SVPWM. La elección del vector nulo determina el patrón de conmutación a usar. Hay pocas opciones: el vector nulo $\mathrm{V}_{0}$ solo, el vector nulo $\mathrm{V}_{7}$ solo, o una combinación de los vectores nulos.

Una técnica popular de SVPWM consiste en alternar el vector nulo en cada ciclo y revertir la secuencia después de cada vector nulo. Esto se denominará Técnica de 7 segmentos simétrica. En la Fig. 4 se muestra la secuencia de conmutación de 7 segmentos convencional del sector I. La secuencia $V_{0}-V_{1}-V_{2}-V_{7}$ se usa en la primera mitad del periodo Ts, y la secuencia $V_{7}-V_{2}-V_{1}-V_{0}$ se usa en la segunda mitad del periodo Ts. Las secuencias son simétricas. La frecuencia de conmutación es la misma que la frecuencia de muestreo del inversor.

Las secuencias de conmutación para los demás sectores siguen el mismo patrón de la Fig. 4. En la 
Tabla III se muestran todas las secuencias de conmutación para cada sector.

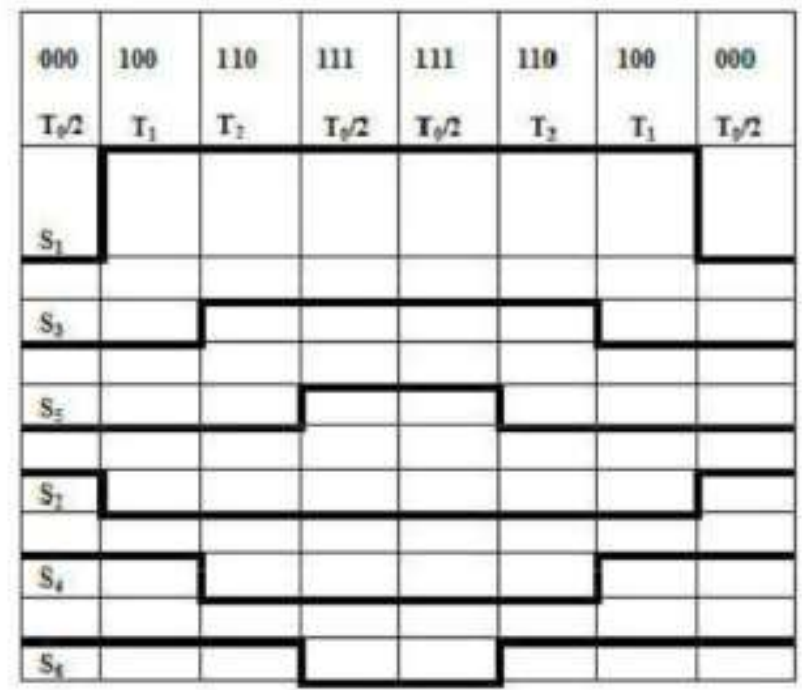

Figura 4: Secuencia de conmutación de 7 segmentos para el sector 1.

Tabla 3: Secuencia de conmutación de los vectores espaciales.

\begin{tabular}{|l|r|r|}
\hline Sectores & \multicolumn{2}{|c|}{ Vectores } \\
\hline & Primera mitad del periódo Ts & Segunda mitad del periódo Ts \\
\hline 1 & $\mathrm{~V}_{0}, \mathrm{~V}_{1}, \mathrm{~V}_{2}, \mathrm{~V}_{7}$ & $\mathrm{~V}_{7}, \mathrm{~V}_{2}, \mathrm{~V}_{1}, \mathrm{~V}_{0}$ \\
\hline 2 & $\mathrm{~V}_{0}, \mathrm{~V}_{3}, \mathrm{~V}_{2}, \mathrm{~V}_{7}$ & $\mathrm{~V}_{7}, \mathrm{~V}_{2}, \mathrm{~V}_{3}, \mathrm{~V}_{0}$ \\
\hline 3 & $\mathrm{~V}_{0}, \mathrm{~V}_{3}, \mathrm{~V}_{4}, \mathrm{~V}_{7}$ & $\mathrm{~V}_{7}, \mathrm{~V}_{4}, \mathrm{~V}_{3}, \mathrm{~V}_{0}$ \\
\hline 4 & $\mathrm{~V}_{0}, \mathrm{~V}_{5}, \mathrm{~V}_{4}, \mathrm{~V}_{7}$ & $\mathrm{~V}_{7}, \mathrm{~V}_{4}, \mathrm{~V}_{5}, \mathrm{~V}_{0}$ \\
\hline 5 & $\mathrm{~V}_{0}, \mathrm{~V}_{5}, \mathrm{~V}_{6}, \mathrm{~V}_{7}$ & $\mathrm{~V}_{7}, \mathrm{~V}_{6}, \mathrm{~V}_{5}, \mathrm{~V}_{0}$ \\
\hline 6 & $\mathrm{~V}_{0}, \mathrm{~V}_{1}, \mathrm{~V}_{6} \mathrm{~V}_{7}$ & $\mathrm{~V}_{7}, \mathrm{~V}_{6}, \mathrm{~V}_{1} \mathrm{~V}_{0}$ \\
\hline
\end{tabular}

\subsection{Implementación en Matlab/Simulink}

La técnica SVPWM presentada anteriormente se ha implementado en el software MATLAB/SIMULINK. En la Fig. 4 se muestra la representación en SIMULINK de la modulación SVPWM para el inversor de dos niveles. Esta representación consta de un inversor de dos niveles implementado mediante IGBT's alimentando una carga trifásica RL y un módulo en Matlab SVPWM dedicado a calcular los tiempos de conmutación de los IGBT's.

\section{Resultados}

La técnica SVPWM presentada se ha implementado en el bloque SVPWM de la Fig. 5 para un periodo de muestreo (conmutación) de $1 \mathrm{~ms}$, con un índice de modulación de 0.8, una tensión de alimentación de 100V y una frecuencia de línea trifásica de $50 \mathrm{~Hz}$.
En la Fig. 6 se muestra la corriente en la carga trifásica, se aprecia un desfase de $120^{\circ}$ y una frecuencia de $50 \mathrm{~Hz}$. Notamos una pequeña distorsión, esta puede ser mejorada aumentando la frecuencia de conmutación. Así también, en la Fig.7 se muestra el voltaje de fase obtenido mediante el algoritmo SVPWM propuesto.

El espectro de la corriente en la carga para el inversor trifásico se muestra en la Fig.8.

Una comparación de la distorsión total armónica (THD) obtenida usando la técnica SVPWM presentada para distintos valores del índice de modulación se muestra en la Tabla IV. En el cálculo del THD se ha considerado los armónicos hasta el orden 25.

\section{Conclusiones}


En este trabajo se ha investigado un enfoque sistemático para lograr una distorsión armónica total (THD) más baja en la corriente de salida del inversor de dos niveles utilizando la modulación de ancho de pulso del vector espacial (SVPWM). Se mostró la representación en SIMULINK de un inversor de dos niveles basado en el esquema SVPWM. La distorsión armónica total de las corrientes trifásicas se determinó a diferentes valores del índice de modulación. Debido a tantas ventajas de SVPWM, se convierte en una buena opción para usar sobre el resto de las técnicas.

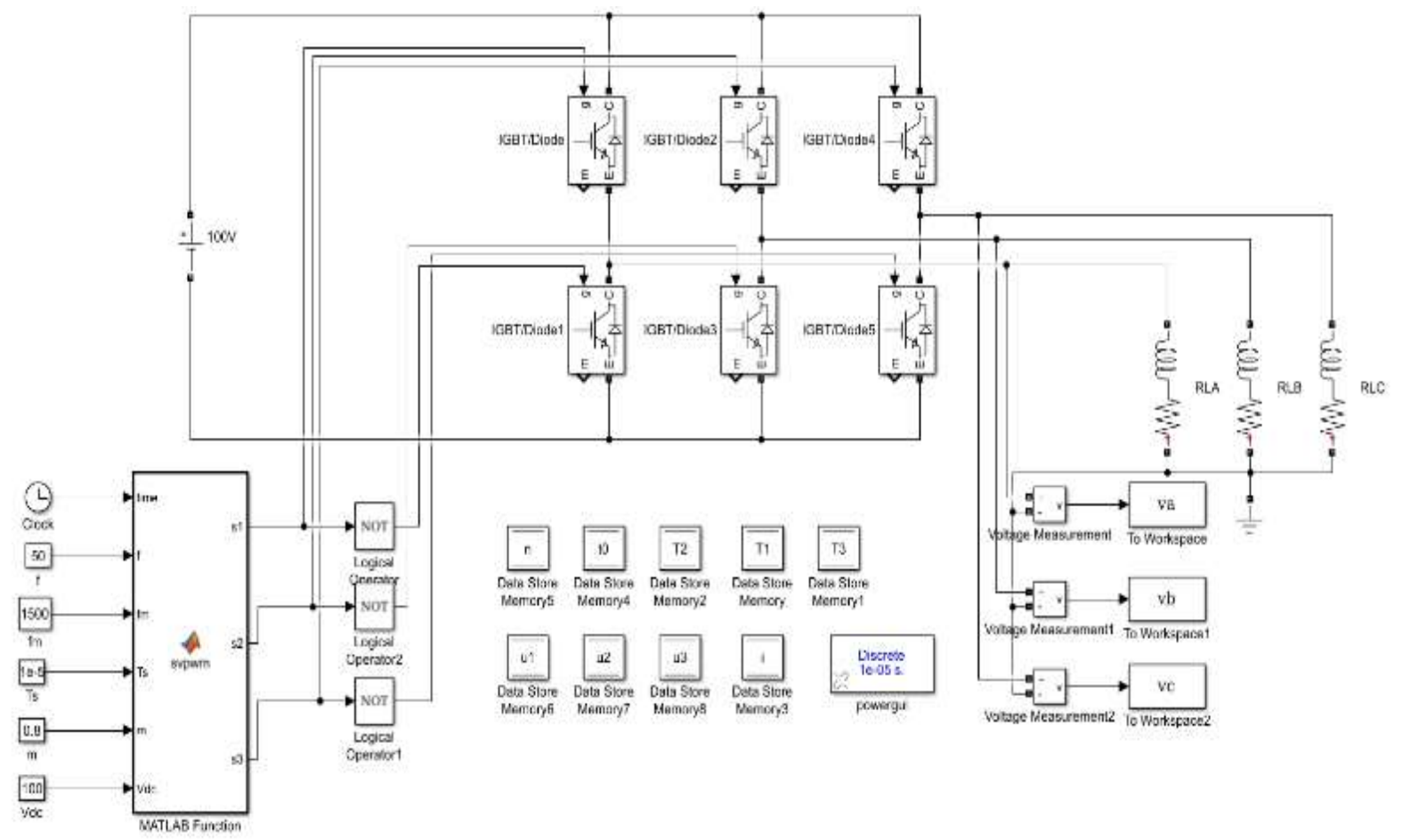

Figura 5: Representación en Simulink de la modulación SVPWM para un inversor de dos niveles.

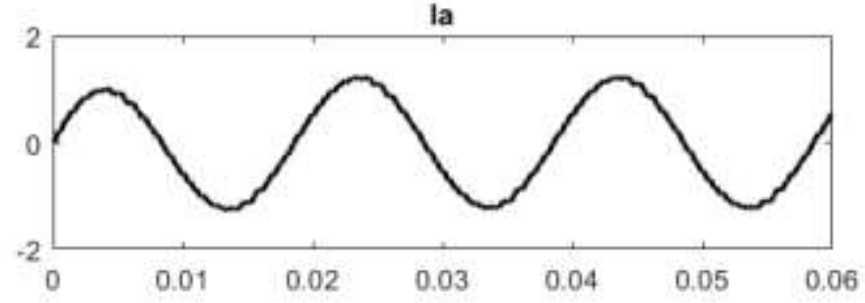

$\mathrm{lb}$

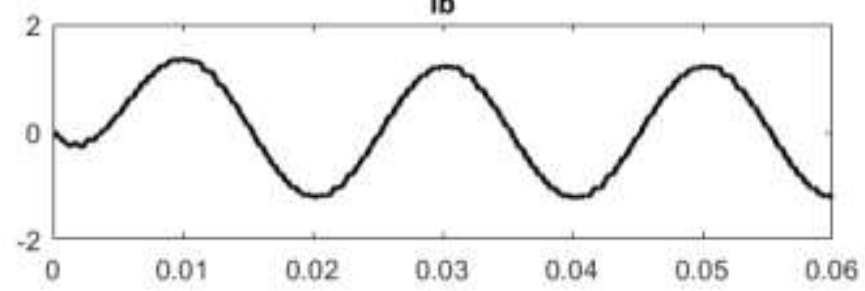

Ic

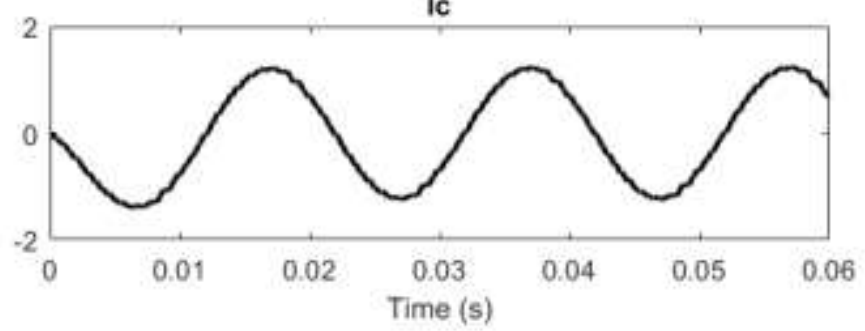

Figura 6: Corriente de la fase trifásica, para una carga de $R=22$ y $L=100 \mathrm{mH}$. 

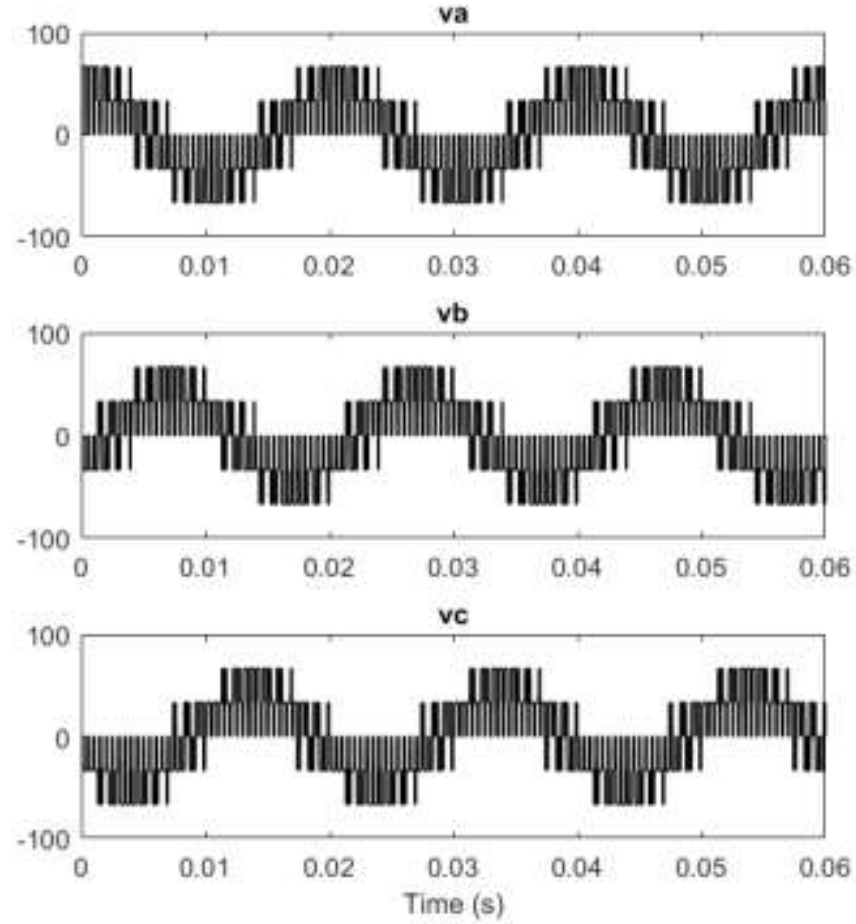

Figura 7: Voltaje de fase para una carga de $R=22$ y $L=100 \mathrm{Mh}$.

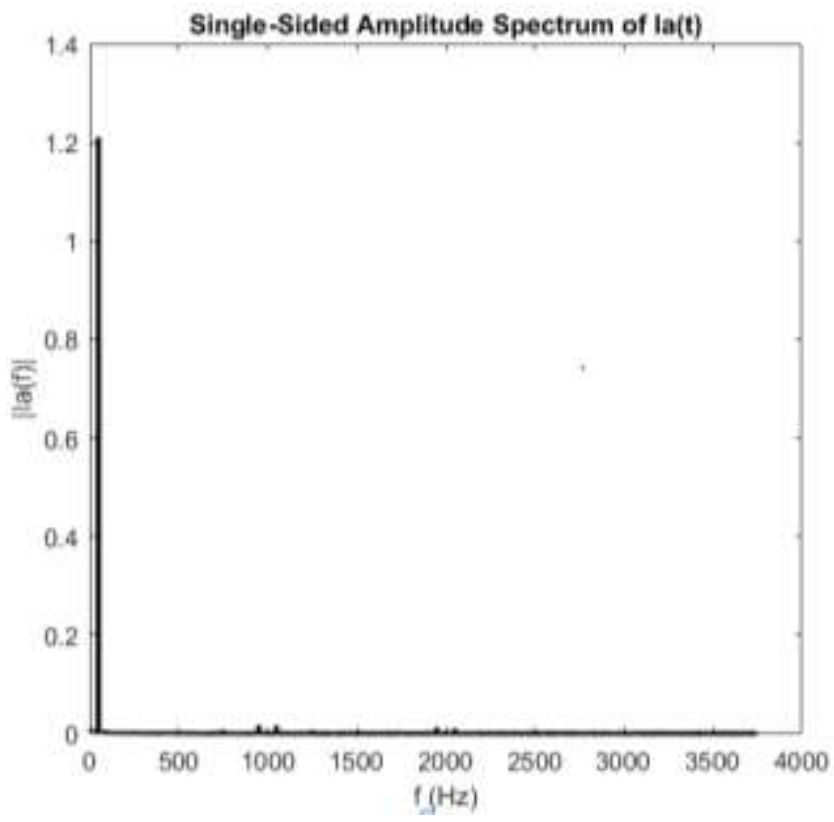

Figura 8: Espectro de la corriente en la carga trifásica.

Tabla 4: Comparativa del THD para distintos valores del Índice de Modulación.

\begin{tabular}{|l|r|}
\hline Índice de modulación & THD (\%) \\
\hline 0.6 & 1.8587 \\
\hline 0.7 & 2.0962 \\
\hline 0.8 & 2.2071 \\
\hline 0.85 & 2.2902 \\
\hline 0.9 & 2.3154 \\
\hline 0.95 & 2.3916 \\
\hline
\end{tabular}




\section{Referencias}

[1] JBV Subrahmanyam, Sankar, International Journal of Engineering and Technology (2011) 15-22.

[2] Ch. Kumar, S. Kamlu and S. Kumar Mishra, ITSI Transactions on Electrical and Electronics Engineering (2013) 15-18.

[3] N. Phung Quang and J. Dittrich, Vector Control of Three-Phase AC Machines, First Edition (Spriger, Berlin, 2008), p.18-53.

\section{E-mail:}

jtisza@gmail.com, jvillegasluis1@gmail.com 